\title{
Nonlinear Sculpturing of Optical Pulses in Fibre Systems
}

\author{
Sonia Boscolo ${ }^{1}$, Ilya Gukov ${ }^{2}$, Alessandro Tonello ${ }^{3}$, Christophe Finot ${ }^{4}$ \\ 1. Aston Institute of Photonic Technologies, School of Engineering and Applied Science, Aston University, Birmingham B4 7ET, UK \\ 2. Moscow Institute of Physics and Technology \& Skolkovo Institute of Science and Technology, Moscow, Russia \\ 3. Laboratoire XLIM, UMR 7252 CNRS - Université de Limoges, Limoges, France \\ 4. Laboratoire Interdisciplinaire Carnot de Bourgogne, UMR 6303 CNRS - Université Bourgogne Franche-Comté, Dijon, France
}

The interplay among the effects of dispersion, nonlinearity and gain/loss in optical fibre systems can be efficiently used to shape the pulses and manipulate and control the light dynamics and, hence, lead to different pulse-shaping regimes [1,2]. However, achieving a precise waveform with various prescribed characteristics is a complex issue that requires careful choice of the initial pulse conditions and system parameters. The general problem of optimisation towards a target operational regime in a complex multi-parameter space can be intelligently addressed by implementing machine-learning strategies. In this paper, we discuss a novel approach to the characterisation and optimisation of nonlinear shaping in fibre systems, which combines numerical simulations of the governing equations to identify the relevant parameters and the machine-learning method of neural networks (NNs) to make predictions across a larger range of the data domain. We illustrate this general method through application to two configurations.

Firstly, we tackle the problem of determining the parameters of pulse shaping systems based on nonlinear pulse propagation in a passive normally dispersive fibre that are required to achieve the generation of pulses with different, simultaneously optimised temporal features. Within our approach, the nonlinear shaping process is reduced to a numerical optimisation problem over the three-dimensional space of normalised fibre length $\xi$, normalised input power $N$, and normalised level of the initial pulse chirp $C$. The intersections of different surfaces provide the means to quickly identify the sets of parameters of interest. We also show that this optimisation problem can be efficiently addressed by application of a regression model based on a $\mathrm{NN}$ algorithm [3].

Secondly, we discuss a new design of a model-locked all-fibre figure-8 (F8) laser employing a nonlinear amplifying loop mirror (NALM) with two active fibre segments and two independently controlled pump-power modules [4]. This laser layout combines the reliability and robustness of conventional F8 lasers with the flexibility of nonlinear-polarisation-evolution lasers, providing access to a variety of generation regimes with a relatively wide adjustment range of the main pulse parameters. In this work, we numerically explore the broad range of operating states of the laser that can be accessed through independent control of the pump powers in the two gain segments and the laser output coupling ratio. We show that the application of an NN-based regression model provides a rapid and precise identification of the output pulse properties that are attainable through variation of the adjustable system parameters, by handling almost instantly the whole parameter space.
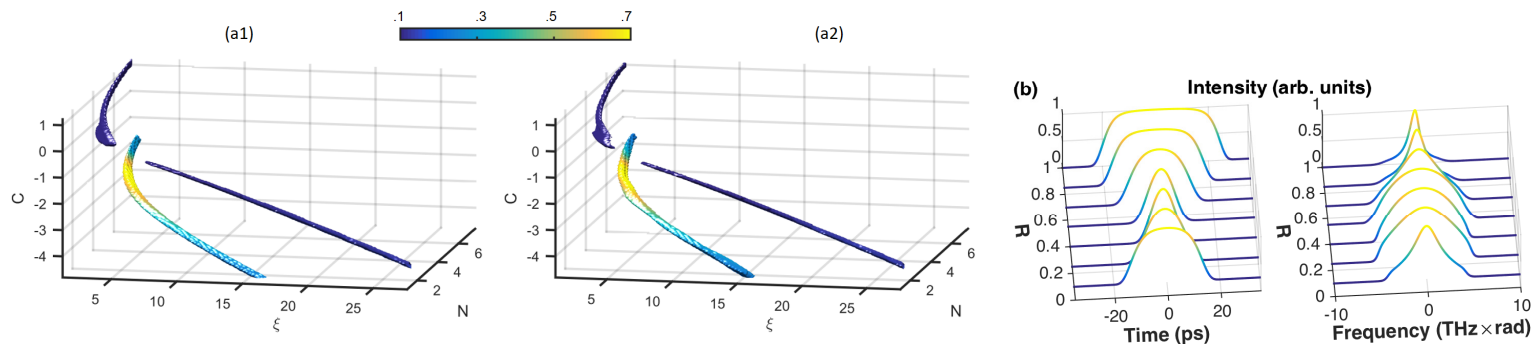

Fig. 1 (a) Pulse shaping in passive normally dispersive fibre. Crossings of parameter surfaces in the three-dimensional parameter space supporting the formation of a parabolic waveform with duration of $10 T_{\text {in }}$. The results obtained from highresolution numerical simulations of the propagation model (subplot 1) are compared with the results generated by the NN regression model (subplot 2). The colormap indicates the Strehl ratio's values of the resulting pulses. (b) Pulse generation in dual-pump NALM mode-locked fibre laser. Evolution of the pulse temporal and spectral intensity profiles with the fractional pump power $R$ for an output coupling factor of 0.75 and a total pump power level delivered to the active fibres of $11.0 \mathrm{~W}$.

Our approach paves the way for alternative approaches to the optimisation of nonlinear dynamics, and can be extended to other complex systems and higher degrees of freedom.

\section{References}

[1] C. Finot, L. Provost, P. Petropoulos, and D. J. Richardson, "Parabolic pulse generation through passive nonlinear pulse reshaping in a normally dispersive two segment fiber device," Opt. Express 15, 852 (2007).

[2] S. Boscolo, A. I. Latkin, and S. K. Turitsyn, "Passive nonlinear pulse shaping in normally dispersive fiber systems," IEEE J. Quantum Electron. 44, 1196 (2008).

[3] C. Finot, I. Gukov, K. Hammani, and S. Boscolo, "Nonlinear sculpturing of optical pulses with normally dispersive fiber-based devices," Opt. Fiber Technol. 45, 306 (2018).

[4] S. Kobtsev, A. Ivanenko, A. Kokhanovskiy, and S. Smirnov, "Electronic control of different generation regimes in mode-locked all-fibre F8 laser," Laser Phys. Lett. 15, 045102 (2018). 